\title{
A New Detached M Dwarf Eclipsing Binary
}

\section{Citation}

Creevey, O. L., G. F. Benedict, T. M. Brown, R. Alonso, P. Cargile, G. Mandushev, D. Charbonneau, et al. 2005. "A New Detached M Dwarf Eclipsing Binary." The Astrophysical Journal 625 (2): L127-30. https://doi.org/10.1086/431278.

\section{Permanent link}

http://nrs.harvard.edu/urn-3:HUL.InstRepos:41417372

\section{Terms of Use}

This article was downloaded from Harvard University's DASH repository, and is made available under the terms and conditions applicable to Other Posted Material, as set forth at http:// nrs.harvard.edu/urn-3:HUL.InstRepos:dash.current.terms-of-use\#LAA

\section{Share Your Story}

The Harvard community has made this article openly available.

Please share how this access benefits you. Submit a story.

\section{Accessibility}


The Astrophysical Journal, 625:L127-L130, 2005 June 1

(C) 2005. The American Astronomical Society. All rights reserved. Printed in U.S.A.

\title{
A NEW DETACHED M DWARF ECLIPSING BINARY
}

\author{
O. L. Creevey, ${ }^{1,2,3}$ G. F. Benedict, ${ }^{4}$ T. M. Brown, ${ }^{1}$ R. Alonso, ${ }^{3}$ P. Cargile, ${ }^{4}$ G. Mandushev, ${ }^{5}$ D. Charbonneau, ${ }^{6}$ \\ B. E. McArthur,${ }^{4}$ W. Cochran, ${ }^{4}$ F. T. O’Donovan, ${ }^{7}$ S. J. Jiménez-Reyes, ${ }^{3}$ J. A. Belmonte, ${ }^{3}$ And D. Kolinski ${ }^{1}$ \\ Received 2005 January 25; accepted 2005 April 21; published 2005 May 11
}

\begin{abstract}
We describe a newly discovered detached $\mathrm{M}$ dwarf eclipsing binary system. This system was first observed by the TrES network during a long-term photometry campaign of 54 nights. Analysis of the folded light curve indicates two very similar components orbiting each other with a period of $1.12079 \pm 0.00001$ days. Spectroscopic observations with the Hobby-Eberly Telescope show the system to consist of two M3e dwarfs in a near-circular orbit. Double-line radial velocity amplitudes, combined with the orbital inclination derived from light-curve fitting, yield $M_{\text {total }}=0.983 \pm 0.007 M_{\odot}$, with component masses of $M_{1}=0.493 \pm 0.003 M_{\odot}$ and $M_{2}=$ $0.489 \pm 0.003 M_{\odot}$. The light-curve fit yields component radii of $R_{1}=0.453 \pm 0.060 R_{\odot}$ and $R_{2}=0.452 \pm$ $0.050 R_{\odot}$. Although a precise parallax is lacking, broadband $V J H K$ colors and spectral typing suggest component absolute magnitudes of $M_{V}(1)=11.18 \pm 0.30$ and $M_{V}(2)=11.28 \pm 0.30$.
\end{abstract}

Subject headings: binaries: close — binaries: eclipsing — stars: individual (TrES-Her0-07621) — stars: late-type

\section{INTRODUCTION}

Although low-mass binary stars are the most abundant stars in the Galaxy (Henry \& McCarthy 1993), their intrinsic faintness inhibits their detection and study. Noncontact eclipsing binary $M$ dwarf systems have great value, as these systems allow us to accurately estimate the most basic stellar parameters: mass and radius. Only four ${ }^{8}$ such systems are known and have been studied in detail: YY Gem (Bopp 1974; Leung \& Schneider 1978), CM Dra (Lacy 1977; Metcalfe et al. 1996; Kozhevnikova et al. 2004), GJ 2069A (Delfosse et al. 1999; Ribas 2003), and OGLE BW03 V038 ${ }^{9}$ (Maceroni \& Montalbán 2004). The observed properties of each of these systems present discrepancies with the theory of low-mass stellar objects; neither the observed mass-radius relation nor the observed massluminosity relation is well represented by existing models (Benedict et al. 2000); see Figure 1. The problem most likely lies in the shortcomings of the physical models, owing to our lack of understanding of the complex atmospheres of such low-mass objects (Baraffe et al. 1998). Enlarging the small existing sample of such systems is therefore desirable, to allow more detailed comparisons between observations and the theory of these ubiquitous, interesting, and complex objects. Here we report a preliminary analysis of a fifth such low-mass eclipsing binary.

\footnotetext{
${ }^{1}$ High Altitude Observatory, National Center for Atmospheric Research, 3450 Mitchell Lane, Boulder 80307, CO; creevey@hao.ucar.edu, timbrown@ hao.ucar.edu, kolinski@hao.ucar.edu.

${ }^{2}$ Universidad de La Laguna, E-38206 La Laguna, Tenerife, Spain.

${ }^{3}$ Instituto de Astrofísica de Canarias, E-38200 La Laguna, Tenerife, Spain; ras@iac.es, sjimenez@iac.es, jba@iac.es.

${ }^{4}$ McDonald Observatory, University of Texas, 1 University Station, C1402, Austin, TX 78712; fritz@astro.as.utexas.edu, p.cargile@mail.utexas.edu,mca@ barney.as.utexas.edu,wdc@shiraz.as.utexas.edu.

${ }^{5}$ Lowell Observatory, 1400 West Mars Hill Road, Flagstaff, AZ 86001; gmand@lowell.edu.

${ }^{6}$ Harvard-Smithsonian Center for Astrophysics, 60 Garden Street, Cambridge, MA 02138; dcharbonneau@cfa.harvard.edu.

${ }^{7}$ California Institute of Technology, 1200 East California Boulevard, Pasadena, CA 91125; francis@caltech.edu.

${ }^{8}$ We refer specifically to binaries for which both components are M dwarfs. However, a number of M dwarf stars whose companion is an F or G MS star have also been detected and studied (e.g., Pont et al. 2005); see Fig. 1.

${ }^{9}$ This is a very close although still detached system.
}

\section{OBSERVATIONS}

\subsection{Photometric Observations}

The recently discovered spectroscopic binary, TrES-Her0-07621 $\left(\alpha=16^{\mathrm{h}} 50^{\mathrm{m}} 20.7, \delta=+46^{\circ} 39^{\prime} 01^{\prime \prime}[\mathrm{J} 2000], V=15.51 \pm 0.08\right)$ was first identified through an analysis of photometric time series from the TrES (Trans-Atlantic Exoplanet Survey) network. This network consists of three telescopes: STellar Astrophysics and Research on Exoplanets ${ }^{10}$ (STARE; Brown \& Charbonneau 1999), Planet Search Survey Telescope ${ }^{11}$ (PSST; Dunham et al. 2004), and Sleuth. ${ }^{12}$ The telescopes are similar in their characteristics, with apertures of $10 \mathrm{~cm}, 2048 \times 2048$ pixel CCD detectors, and fields of view of $6^{\circ} \times 6^{\circ}$.

TrES collects long-term time-series photometry in one filter. The photometry run in question spanned 54 days, beginning in 2003 May 6, and was observed in a band roughly equivalent to Harris $R$ at a cadence of one image every 2 minutes. The images were reduced and calibrated by an automatic package developed specifically for these data. TrES-Her0-07621 was observed by both STARE and PSST, but the latter time series proved significantly noisier. We therefore analyzed only the STARE light curve. The $R$ magnitude is 14.42 , with each point having a formal accuracy of $0.04 \mathrm{mag}$ rms. This light curve contains 8781 data points, obtained in $309.5 \mathrm{hr}$ over 54 days, giving a duty cycle of $23.8 \% .^{13}$

A high signal-to-noise ratio peak in the time series' frequency spectrum at 1.79 cycles per day initiated the study of TrES-Her007621. Folding the star's light curve with a period of 1.1208 days showed it to be an eclipsing binary. The light curve also displays sinusoidal out-of-eclipse variations near the photometric period. The star's infrared colors from the $2 \mathrm{MASS}^{14}$ catalog are quite red

\footnotetext{
${ }^{10}$ Observatorio del Teide, Tenerife, Spain.

${ }^{11}$ Lowell Observatory, Arizona.

${ }^{12}$ Palomar Observatory, California, at http://www.astro.caltech.edu/ ftod/ tres/sleuth.html.

${ }^{13}$ The data are available via the STARE Web site, http://www.hao.ucar.edu/ public/research/stare/stare.html.

${ }^{14}$ Two Micron All Sky Survey: University of Massachusetts and the Infrared Processing and Analysis Center/California Institute of Technology, http:// irsa.ipac.caltech.edu/.
} 


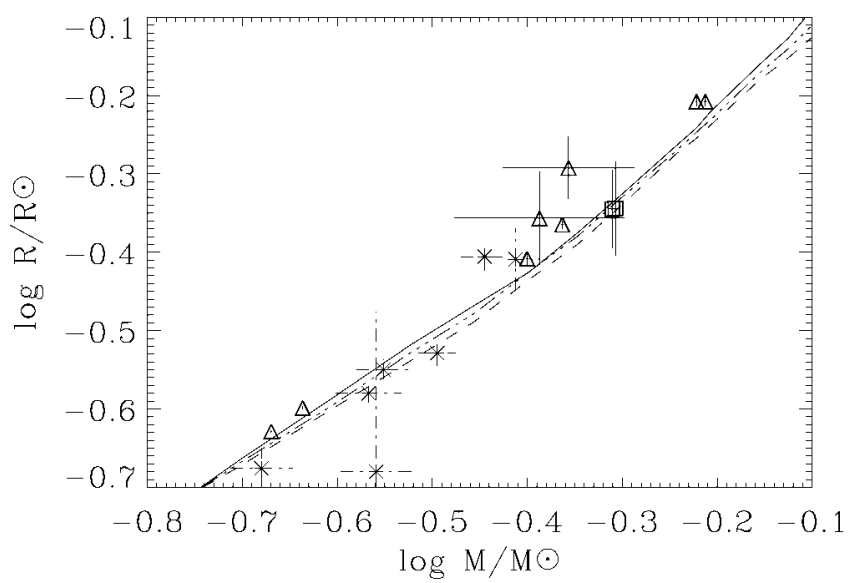

FIG. 1.-Mass-radius relation showing the four other known M dwarf binaries (triangles). Our binary system is represented by squares. We also represent some other M dwarf stars whose companions are F or G MS stars (e.g., Bouchy et al. 2005; Pont et al. 2005). We show theoretical models from Baraffe et al. (1998), indicating an age of 10 (solid line), 5 (dash-dotted line), and $1 \mathrm{Gyr}$ (dashed line), corresponding to $[Z]=0, Y=0.275$.

(Table 1), and the USNO-B ${ }^{15}$ catalog shows a significant proper motion. Taken together, these facts suggested that the object is a binary M dwarf, with substantial levels of magnetic activity driven by the rapid, tidally locked rotation of the component stars; this motivated further study.

\subsection{Spectroscopic Observations}

In 2004 September we obtained spectroscopic observations of TrES-Her0-07621 using the High Resolution Spectrograph (HRS; Tull 1998) on the Hobby-Eberly Telescope (HET). We secured measurements at 4 epochs; each epoch contained three separate exposures taken over approximately $1 \mathrm{hr}$-giving a total of 12 spectra. The analysis was carried out with standard IRAF (Tody 1993) echelle and $r v$ package tools, including fxcorr. We cross-correlate TrES-Her0-07621 with an M2 dwarf (Gl 623) template and extract velocities for both components at four distinct phases. We adopted a radial velocity for the Gl 623 primary of $-29.2 \mathrm{~km} \mathrm{~s}^{-1}$, given the orbital phase at which the template was secured and a systematic velocity, $V_{\text {sys }}=-27.5 \mathrm{~km} \mathrm{~s}^{-1}$, from Marcy \& Moore (1989). The HRS utilizes two CCDs covering the blue and red spectral regions. The data from each chip were analyzed independently, resulting in two velocity estimates. A third velocity estimate was obtained by cross-correlating an artificial $\mathrm{H} \alpha$ emission template with the $\mathrm{H} \alpha$ emission line found in each exposure. Given the large orbital velocities, there was no blending of correlation peaks at any phase. The three velocities (blue, red, $\mathrm{H} \alpha$ ) are obviously not independent determinations but do provide an estimate of our internal error.

\section{ANALYSIS}

Figure 2 shows the component velocities plotted against photometric phase, while Figure 3 (top panel) shows the folded photometric light curve. It is evident from the nearly symmetrical and sinusoidal radial velocity variation and from the highly symmetrical light curve that the orbit is nearly circular and that the component masses and surface brightnesses are similar.

An initial period analysis of the entire STARE light curve using the technique of phase dispersion minimization refined the pho-

${ }^{15}$ SIMBAD, operated at CDS, Strasbourg, France, and the NASA/IPAC Extragalactic Database (NED), supported by JPL, California Institute of Technology, http://www.nofs.navy.mil/data/fchpix.
TABLE 1

CATAlog Information

\begin{tabular}{|c|c|c|}
\hline Parameter & Binary & Neighbor \\
\hline ....... & 11.773 & 13.487 \\
\hline$\ldots \ldots \ldots \ldots \ldots \ldots \ldots$ & 11.137 & 12.863 \\
\hline$\ldots \ldots \ldots \ldots \ldots$ & 10.880 & 12.615 \\
\hline$J-K \ldots \ldots \ldots \ldots \ldots \ldots$ & 0.893 & 0.872 \\
\hline$\mu_{\alpha} \ldots \ldots \ldots \ldots \ldots \ldots \ldots$ & $-2 \pm 8$ & $-26 \pm 14$ \\
\hline$\mu_{\delta} \ldots \ldots \ldots \ldots \ldots \ldots \ldots$ & $+30 \pm 3$ & $+28 \pm 7$ \\
\hline
\end{tabular}

NoTE.-Color information from 2MASS, significant proper motion data from USNO-B (units of mas $\mathrm{yr}^{-1}$ ).

tometric period to $1.1209 \pm 0.0006$ days. We predicted and then observed an eclipse on 2004 May 14 using the $1.2 \mathrm{~m}$ telescope at the Fred L. Whipple Observatory, Arizona, using Sloan filters $r, i$, and $z$. The long time base provided by this observation allowed us to refine the photometric period. By fitting the light curves during (21) eclipse times (we included only totally observed eclipses) to parabolas, we determined all the times of minimum light (center of eclipse) with corresponding error. For the eclipse observed on 2004 May 14, we only used the time of minimum light from the $r$ filter. We also used observations from IAC80 (see below). Using the bootstrap method, we refined the period to $1.12079 \pm 0.00001$, corresponding to a precision of $1 \mathrm{~s}$. The epoch of secondary minimum, $T_{0}$, was meanwhile determined to be 2,453,139.749509 (HJD) \pm 0.000075 .

TrES-Her0-07621 has a stellar neighbor at a distance of 8", close enough that the two objects are blended in our STARE observations (STARE has a pixel size of about $11^{\prime \prime}$ ). Observations in $R$ and $I$ Johnson filters using the IAC80 at Observatorio del Teide on 2004 August 30 provided a more realistic picture of the depth of one of the eclipses, while also allowing us to confirm the photometric period. We measured the point-spread function of both the binary and the neighbor using all five images outside of the eclipse. From these we derived the $R$ fractional flux contribution from this companion star of $0.19 \pm 0.04$. Because the companion star is also quite red (Table 1), the flux should be similar (to within the error) in both Johnson and Harris $R$ filters, and so we can use this number to analyze the STARE time series. Measurement of the contamination of the eclipse signal from TrES-

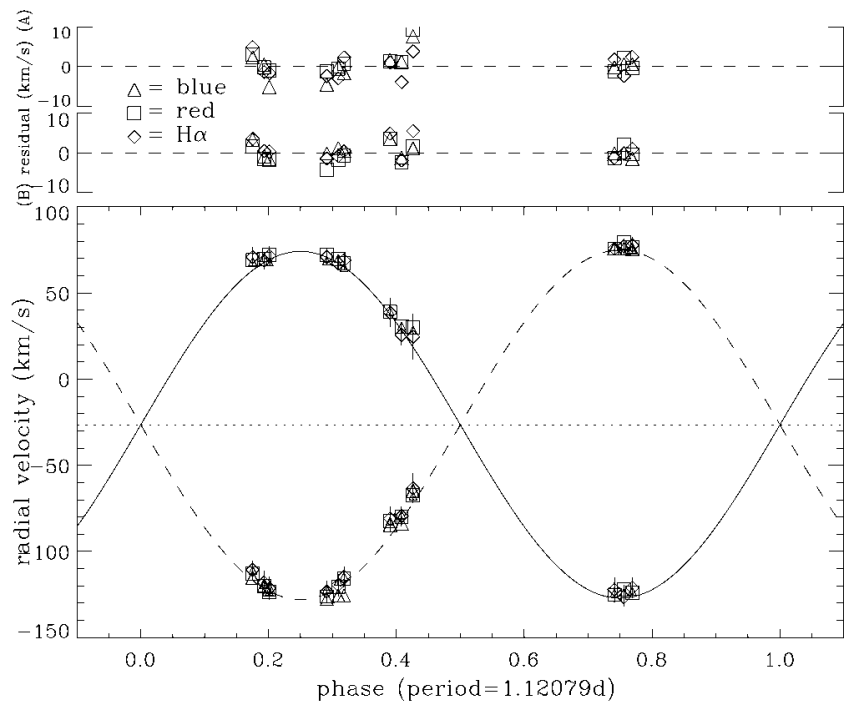

FIG. 2.-Phased radial velocity curve of TrES-Her0-07621 with residuals; data observed by the HET. A total of four nights were obtained; the system was observed for $1 \mathrm{hr}$ every night at 20 minutes per exposure. The Doppler displacement was measured in two different wavelengths regions and at $\mathrm{H} \alpha$. 


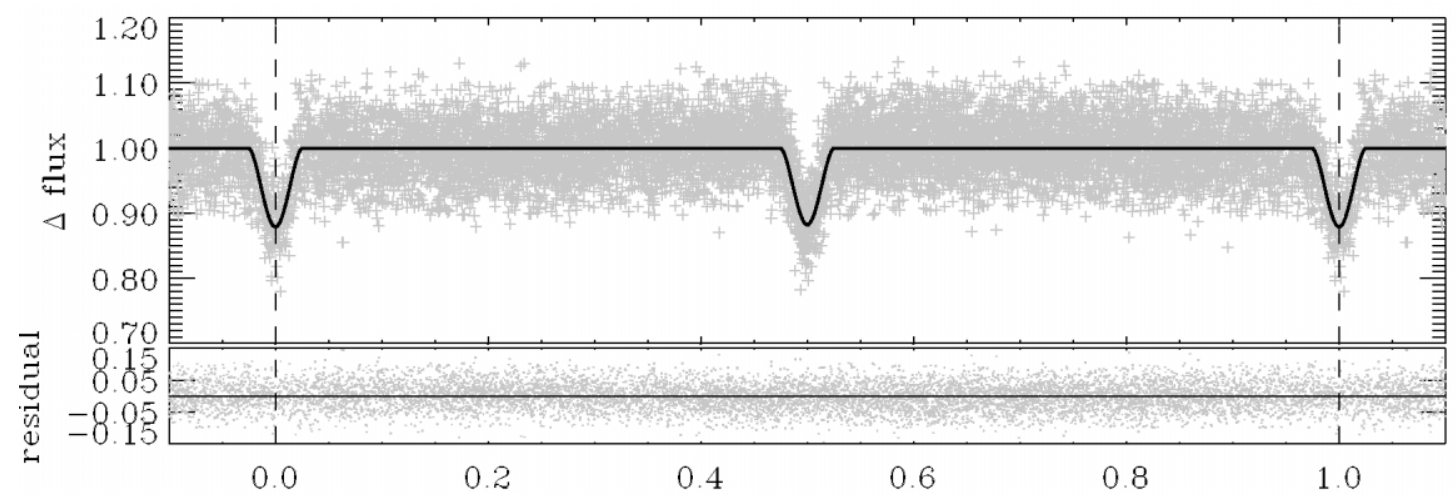

FIG. 3.-Observed light-curve and fitting results. The top panel shows the reconstructed light curve (solid line) using the parameters obtained by fitting the observed light curve without the out-of-eclipse variations (small crosses; see $\S 4$ ). We have indicated phase $=0$ and 1 (center of secondary eclipse) by dashed lines. The bottom panel shows the residuals (dots), with the solid line representing the rms of residuals.

Her0-07621 by the companion star is important, because it must be accounted for when fitting the time-series data to estimate the stellar radii. This neighbor also has a proper motion that is similar in magnitude and direction to that of TrES-Her0-07621, indicating the possibility that TrES-Her0-07621 is at least a triple system, with the eclipsing pair of stars accompanied by a third $\mathrm{M}$ dwarf at a distance of hundreds of AU.

Adopting the photometric period as the orbital period and introducing its associated error, we fit all 36 radial velocities (blue, red, $\mathrm{H} \alpha$ ) with a Keplerian model using GaussFit (Jefferys et al. 1988). The model is similar to that used in McArthur et al. (2004). We assume an eccentricity $e$ of 0 . The resulting radial velocity semiamplitudes are $K_{1}=100.54 \pm 0.31 \mathrm{~km} \mathrm{~s}^{-1}$ and $K_{2}=101.29 \pm$ $0.31 \mathrm{~km} \mathrm{~s}^{-1}$, giving $\left(M_{1}+M_{2}\right) \sin ^{3} i=0.9547 \pm 0.0062 M_{\odot}$ and $M_{1} / M_{2}=1.0075 \pm 0.0044$. A formal solution including eccentricity $(e=0.006 \pm 0.002)$ provided a better solution, reducing $\chi^{2}$ by $8 \%$, while reducing the number of degrees of freedom by $3 \%$. However, we constrain $e=0$ for this analysis.

We developed a $\chi^{2}$ minimization algorithm to estimate orbital parameters from the light curve, ignoring any variations between eclipses (Fig. 3). The input parameters are period $P$, component masses $M_{1}$ and $M_{2}$, limb-darkening coefficients ( 0.7 ; Claret 1998, Table 7), and the light from a third nearby star as a fraction of the total light of the system $(0.19 \pm 0.04)$. The code solves for both radii $R_{1}$ and $R_{2}$, the effective temperature ratio $T_{2} / T_{1}$, the center of minimum eclipse $T_{0}$, and inclination $i$. Figure 3 shows the resulting fit to the data.

The initial estimates for $R_{1}, R_{2}, T_{2} / T_{1}$, and $i$ were derived from two-dimensional $\chi^{2}$ contour plots (while keeping the other two parameters fixed). These contour plots presented high correlations between the two radii, constraining their sum while insensitive to their difference, and between radius $\left(R_{1}\right.$ or $\left.R_{2}\right)$ and inclination; larger radius implies smaller inclination. $T_{2} / T_{1}$ was uncorrelated to both radii and inclination, so its error is given

TABLE 2

System Parameters

\begin{tabular}{lc}
\hline \hline Parameter & \multicolumn{1}{c}{ Value } \\
\hline$P$ (days) $\ldots \ldots \ldots$ & $1.12079 \pm 0.00001$ \\
$P$ (yr) $\ldots \ldots \ldots \ldots$ & $0.00306861 \pm 0.00000036$ \\
$T_{0}(\mathrm{HJD}) \ldots \ldots \ldots$ & $2,453,139.749509 \pm 0.00075$ \\
$M_{T}\left(M_{\odot}\right) \ldots \ldots \ldots$ & $0.983 \pm 0.007$ \\
$a(\mathrm{AU}) \ldots \ldots \ldots \ldots$ & $0.01047 \pm 0.00002$ \\
$a\left(R_{\odot}\right) \ldots \ldots \ldots \ldots$ & $2.251 \pm 0.005$ \\
$i(\mathrm{deg}) \ldots \ldots \ldots \ldots$ & $83.12 \pm 0.30$ \\
$\gamma\left(\mathrm{km} \mathrm{s}^{-1}\right) \ldots \ldots$. & $-26.5 \pm 0.3$ \\
$T_{B} / T_{A} \ldots \ldots \ldots \ldots$ & $0.97 \pm 0.02$ \\
\hline
\end{tabular}

by the corresponding value of this parameter at $\chi^{2}+\sigma$ (Press et al. 1986, § 14.5) in the direction of its axis. However, because the other parameters are obviously not independent, $R_{1}$ and $R_{2}$ for example, the error spanned the range of radii where the contour value is $\chi^{2}+\sigma$ (the full range error ellipsoid).

Even with the component masses determined, in the absence of a $T_{\text {eff }}$ measurement we require the component absolute magnitudes to place these stars on the mass-luminosity relation. From the TrES data, calibrated using stars within $1^{\circ}$ that have measured $V$ magnitudes from SIMBAD, we obtain a $V$-band apparent magnitude of $15.51 \pm 0.08$ for the combined three-star system, giving $V-K=4.63 \pm 0.10$ (Table 1). Assuming a wavelength-independent relative flux, we estimate $\Delta V_{\mathrm{AB}-\mathrm{C}}=1.72$ from difference $J, H$, and $K$ magnitudes (between the neighbor and the binary). We can also estimate $\Delta V_{\mathrm{A}-\mathrm{B}}$ (between the binary components) = $0.1 \pm 0.05$, based on the derived temperature and radii differences. Taking all of the above into account, we estimate the component magnitudes of $V_{\mathrm{A}}=16.37 \pm 0.1, V_{\mathrm{B}}=16.56 \pm 0.1$, and $V_{\mathrm{C}}=$ $17.43 \pm 0.1$, where $\mathrm{C}$ is the stellar neighbor.

From the Hawley et al. (2002) color-spectral type relations, we estimate an M3 spectral type for each component. From the Hawley et al. $M_{J}$-spectral type relationships, we obtain component absolute magnitudes of $M_{V}=11.18,11.28 \pm 0.3$. Accepting this estimate of the luminosities, the distance modulus is $\mu(\sim 16.4-11.2) \sim 5.2$, corresponding to $d \sim 110$ pc. For this nearby system, we have assumed no absorption $\left(A_{V}=0\right)$. We also use the radii and effective temperature (Table 3 ) to determine luminosities, differentially with respect to the Sun (e.g., Benedict et al. 2003). With bolometric corrections as a function of temperature, from Flower (1996) we obtain an average $d=118 \pm 13 \mathrm{pc}$ for the two components.

\section{RESULTS AND COMMENTS}

Using all the derived parameters and errors, we refit the light curve using our code, and we tested these results with the code

TABLE 3

Component Parameters

\begin{tabular}{ccc}
\hline \hline Parameter & $\mathrm{A}$ & $\mathrm{B}$ \\
\hline$M\left(M_{\odot}\right) \ldots \ldots \ldots$. & $0.493 \pm 0.003$ & $0.489 \pm 0.003$ \\
$M_{V} \ldots \ldots \ldots \ldots \ldots$ & $11.18 \pm 0.30$ & $11.28 \pm 0.30$ \\
$K\left(\mathrm{~km} \mathrm{~s}^{-1}\right) \ldots \ldots$ & $100.54 \pm 0.31$ & $101.29 \pm 0.31$ \\
$R\left(R_{\odot}\right) \ldots \ldots \ldots$. & $0.453 \pm 0.060$ & $0.452 \pm 0.050$ \\
$T_{\text {eff }}{ }^{\mathrm{a}}(\mathrm{K}) \ldots \ldots \ldots$ & 3500 & 3395 \\
\hline
\end{tabular}

${ }^{a}$ The component A temperature is based on that expected of an M3 V star (Cox 2000). 
Nightfall $^{16}$ (see below). Both codes give similar results, their difference being within the error bars. Tables 2 and 3 summarize the results.

Our code does not allow for stellar spots, so we subtracted a smooth function (by a Fourier technique) in order to remove the out-of-eclipse variations, making a rectified light curve. We also constrain $e=0$. The top panel of Figure 3 shows the synthetic light curve (solid line) corresponding to the model fit (our code) of the folded light curve (small crosses). Phase $=0$ corresponds to the secondary eclipse. The bottom panel shows the residuals of the fit. The residuals show no variation as a function of phase, indicating an adequate model fit.

Because our code is unable to account for spot variability, we inspected the residuals after subtracting the model fit from the unrectified light curve. These residuals also showed no evidence of eclipses. We also fit this unrectified light curve in order to find $R_{1}, R_{2}, i, T_{0}$, and $T_{2} / T_{1}$. The results varied slightly from those for the rectified light curve but stayed within the error bars (Table 3).

Our original (unrectified) photometric light curve contains nonuniform out-of-eclipse variations. Binary systems such as TrES-Her0-07621 are often magnetically active (e.g., Strassmeier et al. 1993). While tidal effects may be important, these nonuniform variations are most likely explained by star spots. We used Nightfall to model our unrectified light curve, because this code allows for the presence of spots on each of the components. Our derived parameters were used as inputs, and we attempted to solve for the longitude, latitude, and radii of the spot(s). There was no unique solution; many combinations of these spot pa-

${ }^{16}$ See http://www.lsw.uni-heidelberg.de/users/rwichman/Nightfall.html. rameters could compensate for the out-of-eclipse variations, although they always presented a $180^{\circ}$ longitude difference. This preferred longitude difference has also been observed in other active binary systems (see, e.g., Henry et al. 1995). The presence of spots can have a significant effect on the accuracy of the derived parameters, such as inclination, temperature, and radii (Torres \& Ribas 2002 discuss this for the case of YY Gem). Additional observations, photometry in particular, will be necessary to increase the precision of the radius estimates as well as to learn more about the magnetic behavior of the stars. This could then provide a link toward a better understanding of the physical processes of these low-mass objects.

We thank Hectór Vazquez Ramio (observations on IAC80) and the operating staff for STARE. The IAC80 and STARE are operated by the Instituto de Astrofísica de Canarias in the Spanish Observatorio del Teide. We thank Mark Everett for observations on the 48 inch $(1.2 \mathrm{~m})$ telescope at Fred L. Whipple Observatory on Mount Hopkins, Arizona, operated by the Harvard-Smithsonian Center for Astrophysics. We also thank Rainer Wichmann for the use of the program for the light-curve synthesis, Nightfall. Support for this work was provided by NASA through grants GO-09408 and GO-09407 and the Space Telescope Science Institute, which is operated by the Association of Universities of Research in Astronomy, Inc., under NASA contract NAS526555. The Hobby-Eberly Telescope (HET) is a joint project of the University of Texas at Austin, Pennsylvania State University, Stanford University, Ludwig-Maximilians-Universität München, and Georg-August-Universität, Göttingen. We thank the HET resident astronomers and telescope operators. We thank the referees for their constructive comments.

\section{REFERENCES}

Baraffe, I., Chabrier, G., Allard, F., \& Hauschildt, P. H. 1998, A\&A, 337, 403 Benedict, G. F., McArthur, B. E., Franz, O. G., Wasserman, L. H., \& Henry, T. J. 2000, AJ, 120, 1106

Benedict, G. F., et al. 2003, AJ, 126, 2549

Bopp, B.W. 1974, ApJ, 193, 389

Bouchy, F., Pont, F., Melo, C., Santos, N. C., Mayor, M., Queloz, D., \& Udry, S. 2005, A\&A, 431, 1105

Brown, T. M., \& Charbonneau, D. 1999, BAAS, 31, 1534

Claret, A. 1998, A\&A, 335, 647

Cox, A. N. 2000, Allen's Astrophysical Quantities (4th ed.; New York: AIP)

Delfosse, X., Forveille, T., Mayor, M., Burnet, M., \& Perrier, C. 1999, A\&A, 341, L63

Dunham, E. W., Mandushev, G. I., Taylor, B. W., \& Oetiker, B. 2004, PASP, 116,1072

Flower, P. J. 1996, ApJ, 469, 355

Hawley, S. L., et al. 2002, AJ, 123, 3409

Henry, G. W., Eaton, J. A., Hamer, J., \& Hall, D. S. 1995, ApJS, 97, 513

Henry, T. J., \& McCarthy, D. W. 1993, AJ, 106, 773

Jefferys, W. H., Fitzpatrick, M. J., \& McArthur, B. E. 1988, Celest. Mech., 41,39
Kozhevnikova, A. V., Kozhevnikov, V. P., Zakharova, P. S., Polushina, T. S., \& Svechnikov, M. A. 2004, Astron. Rep., 48, 826

Lacy, C. 1977, ApJ, 218, 444

Leung, K., \& Schneider, D. 1978, AJ, 83, 618

Maceroni, C., \& Montalbán, J. 2004, A\&A, 426, 577

Marcy, G. W., \& Moore, D. 1989, ApJ, 341, 961

McArthur, B. E., et al. 2004, ApJ, 614, L81

Metcalfe, T. S., Mathieu, R. D., Latham, D. W., \& Torres, G. 1996, ApJ, 456, 356

Pont, F., Bouchy, F., Melo, C., Santos, N. C., Mayor, M., Queloz, D., \& Udry, S. 2005, A\&A, submitted (astro-ph/0501615)

Press, W. H., Flannery, B. P., Teukolsky, S. A., \& Vetterling, W. T. 1986, Numerical Recipes (Cambridge: Cambridge Univ. Press)

Ribas, I. 2003, A\&A, 398, 239

Strassmeier, K. G., Hall, D. S., Fekel, F. C., \& Scheck, M. 1993, A\&AS, 100, 173

Torres, G., \& Ribas, I. 2002, ApJ, 567, 1140

Tody, D. 1993, in ASP Conf. Ser. 52, Astronomical Data Analysis Software and Systems II, ed. R. J. Hanisch, R. J. V. Brissenden, \& J. Barnes (San Francisco: ASP), 173

Tull, R. G. 1998, Proc. SPIE, 3355, 387 